\title{
Imputation of faulty magnetic sensors with coupled Bayesian and Gaussian processes to reconstruct the magnetic equilibrium in real time ${ }^{\mathrm{a})}$
}

\author{
Semin Joung, ${ }^{1,}$ b) Jaewook Kim, ${ }^{1}$ Sehyun Kwak, ${ }^{1,2}$ Kyeo-reh Park, ${ }^{1}$ S.H. Hahn, ${ }^{3}$ H.S. Han, ${ }^{3}$ H.S. Kim, ${ }^{3}$ \\ J.G. Bak, ${ }^{3}$ S.G. Lee, ${ }^{3}$ and Y.-c. Ghim ${ }^{1, c)}$ \\ ${ }^{1)}$ Department of Nuclear and Quantum Engineering, KAIST, Daejeon 34141, Republic of Korea \\ ${ }^{2)}$ Max-Planck-Institut für Plasmaphysik, Teilinstitut Greifswald, D-17491 Greifswald, Germany \\ ${ }^{3)}$ National Fusion Research Institute, Daejeon 34133, Republic of Korea
}

(Dated: 25 June 2018)

\begin{abstract}
A Bayesian with GP(Gaussian Process)-based numerical method to impute a few missing magnetic signals caused by impaired magnetic probes during tokamak operations is developed such that the real-time reconstruction of magnetic equilibria, whose performance strongly depends on the measured magnetic signals and their intactness, are affected minimally. Likelihood of the Bayesian model constructed with the Maxwell's equations, specifically Gauss's law for magnetism and Ampère's law, results in infinite number of solutions if two or more magnetic signals are missing. This undesirable characteristic of the Bayesian model is remediated by coupling the model with the Gaussian process. Our proposed numerical method infers nine non-consecutive missing magnetic signals correctly in less than 1 msec suitable for real-time reconstruction of magnetic equilibria during tokamak operations.
\end{abstract}

\section{INTRODUCTION}

Magnetic pick-up coils installed on magnetic confinement devices such as tokamaks and stellarators in addition to Rogowski and flux loop coils provide magnetic information such that high temperature fusion-grade plasmas can be controlled in real time and that magnetic equilibria can be reconstructed for data analyses. Neural networks also have been developed to provide the positions of $\mathrm{X}$-point and plasma boundary in real time ${ }^{1,2}$ where input signals to the networks are magnetic signals. Therefore, integrity of the magnetic signals is of paramount importance. As magnetic probes are subject to impairment during plasma operations, faulty plasma operations and incorrect data analyses can be caused by missing magnetic signals. For the case of neural networks trained with full sets of magnetic signals, even a single missing signal may cause the networks not to work properly. $^{3}$

We present how one can numerically infer, thus impute, missing magnetic signals in real time based on a Bayes' model $^{4}$ coupled with the Gaussian Process ${ }^{5}$ (GP). Likelihood is constructed using Gauss's law for magnetism and Ampère's law and ensuring the consistency with the measured data. A couple of algorithms to detect faulty magnetic sensors in real time have been developed, ${ }^{6,7}$ and an inference method for just one faulty signal has also been proposed. ${ }^{7}$ Our proposed method in this work is tested with up to nine non-consecutive missing magnetic probe signals installed on KSTAR. ${ }^{8}$ We find that the method

\footnotetext{
a) Contributed paper published as part of the Proceedings of the 22nd Topical Conference on High-Temperature Plasma Diagnostics, San Diego, California, April, 2018.

b) smjoung@kaist.ac.kr

c)ycghim@kaist.ac.kr
}

infers the correct values in less than $1 \mathrm{msec}$ on a typical personal computer. Then, a full set of raw data, i.e., inferred ones together with measured ones, can be passed for real-time EFIT reconstruction ${ }^{9,10}$ and neural networks.

Detailed descriptions on how we generate the likelihood and estimate the maximum a posteriori of the Bayes' model and how well the model infers the missing values as well as its limitation are provided in section II A. The limitation on the Bayes' model motivates us to use the GP discussed in section II B which also has a certain drawback. In section II C, we present improved performance, i.e., resolving the defects of the Bayes' model and the GP while retaining their advantages, achieved by coupling the Bayes' model with the GP. To test our proposed method we assume that the intact magnetic signals are missing and compare the measured signals with the inferred values. Our conclusion is presented in section III.

\section{IMPUTATION SCHEME}

\section{A. Based on Bayes' model}

Magnetic probes, ${ }^{11}$ depicted in Fig. 1(a) as the blue dots with the probe numbers, installed on KSTAR at a certain toroidal location measure tangential $\left(B_{t}\right)$ and normal $\left(B_{n}\right)$ components of the magnetic fields with respect to the wall. Missing tangential components are inferred using Ampère's law with the measured plasma currents by Rogowski coils, i.e., $\nabla \times \vec{B}=\mu_{0} \vec{J}$ neglecting $\partial \vec{E} / \partial t$ term based on a usual magnetohydrodynamic assumption, ${ }^{12}$ and missing normal components using Gauss's law for magnetism, i.e., $\nabla \cdot \vec{B}=0$.

With the Amperian loop, the blue line connecting the blue dots shown in Fig. 1(a), the tangential components 


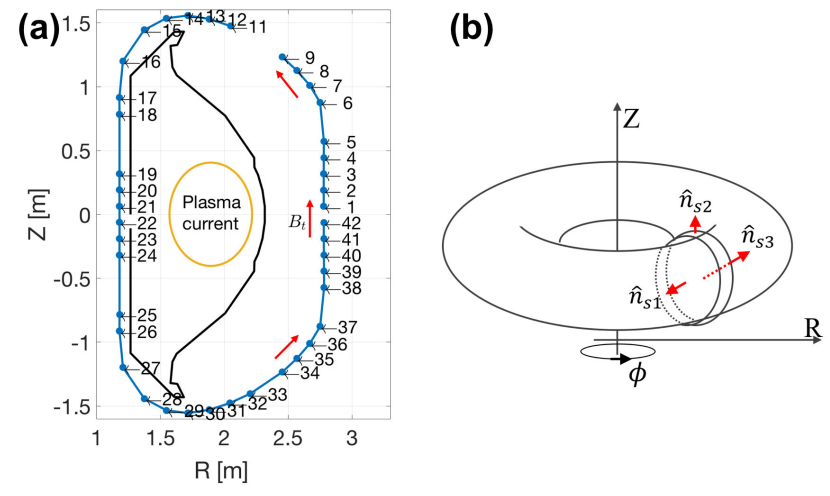

FIG. 1: Schematics of (a) the Amperian loop (blue line connecting blue dots) for $\nabla \times \vec{B}=\mu_{0} \vec{J}$ and (b) the pancake-shaped Gaussian surface with three surfaces $s_{1}$, $s_{2}$ and $s_{3}$ for $\nabla \cdot \vec{B}=0$. Blue dots with the numbers in (a) indicate the magnetic probes. ${ }^{11}$

of the magnetic signals $B_{t}$ must approximately satisfy

$$
\begin{aligned}
& \mu_{0} I_{p}=\oint_{L} \vec{B} \cdot d \vec{l} \approx \oint_{L}\left(B_{t}^{\mathrm{MP}}-B_{t}^{\mathrm{PF}}\right) d l \\
& \approx\left[\sum_{m=1}^{N_{m}} \Delta l_{m}^{*}\left(B_{t, m}^{* \mathrm{MP}}-B_{t, m}^{* \mathrm{PF}}\right)+\right. \\
&\left.\sum_{i=1}^{N_{i}} \Delta l_{i}\left(B_{t, i}^{\mathrm{MP}}-B_{t, i}^{\mathrm{PF}}\right)\right] \\
& \boldsymbol{\lambda}^{* T}\left(\boldsymbol{B}_{t}^{* \mathrm{MP}}-\boldsymbol{B}_{t}^{* \mathrm{PF}}\right)+ \\
& \boldsymbol{\lambda}^{T}\left(\boldsymbol{B}_{t}^{\mathrm{MP}}-\boldsymbol{B}_{t}^{\mathrm{PF}}\right),
\end{aligned}
$$

where $I_{p}$ is the total plasma current assuming that the effect of transient eddy currents is negligible ${ }^{13}$ which, in general, is acceptable at least during a flat-top phase. $B_{t}^{\mathrm{MP}}$ and $B_{t}^{\mathrm{PF}}$ are the tangential components of the magnetic fields measured by the magnetic probes (MP) and induced by the poloidal field (PF) coils, respectively. Note that KSTAR has $14 \mathrm{PF}$ coils, and their contributions are not perfectly canceled out due to change of integral form to a summation. Therefore, we remove the PF coil contributions. $m$ and $i$ are the indices for the missing and the intact magnetic signals; whereas $N_{m}$ and $N_{i}$ are the total numbers of the missing and the intact signals, respectively. $\Delta l$, an approximation of $d l$, denotes the segment distance between the magnetic probes, i.e., the distance between the consecutive probe numbers in Fig. 1(a). $\Delta l$ is different for different probes as can be seen in Fig. 1(a). Superscripted asterisk means the missing magnetic signal. The last line in Eq. (1) is just a reformulation of the second line using the vector notations, i.e., $\boldsymbol{\lambda}^{(*)}=\left\{\Delta l_{i(m)}^{(*)}\right\}$ and $\boldsymbol{B}_{t}^{(*)}=\left\{B_{t, i(m)}^{(*)}\right\}$. Moret et $a l .{ }^{14}$ has used Eq. (1) to obtain plasma currents in TCV tokamak; whereas we apply the same idea to obtain the missing magnetic signals based on the plasma currents measured by Rogowski coils.

For the normal components of the magnetic signals, we utilize the pancake-shaped Gaussian surface as depicted in Fig. 1(b) consisting of three surfaces $s_{1}, s_{2}$ and $s_{3}$. We force the Gaussian surface to be flat enough, so that the magnetic fluxes through the surfaces of $\hat{n}_{s_{1}}$ and $\hat{n}_{s_{3}}$ cancel each other as $\hat{n}_{s_{1}} \cdot \hat{n}_{s_{3}}=-1$, where $\hat{n}$ is a unit normal vector. Then, $\nabla \cdot \vec{B}=0$ can be written as

$$
0=\oint_{s_{1}+s_{2}+s_{3}} \vec{B} \cdot d \vec{S} \approx \int_{s_{2}} B_{n} d A \approx \Delta w \int_{L} B_{n} d l,
$$

where $d A(=\Delta w d l)$ is the differential area normal to the surface $s_{2}$ (parallel to $B_{n}$ ) with $\Delta w$ being the thickness of the Gaussian surface. $d l$ is the differential length encompassing the minor radius (or the poloidal cross-section) and essentially same as the blue line in Fig. 1(a). Since $\Delta w \neq 0$, we have, again with the vector notations,

$$
0=\int_{L} B_{n} d l \approx \boldsymbol{\lambda}^{* T} \boldsymbol{B}_{n}^{*}+\boldsymbol{\lambda}^{T} \boldsymbol{B}_{n}
$$

Assuming that the noise in magnetic signals is Gaussian, the likelihood is

$$
\begin{aligned}
p\left(\boldsymbol{B}_{\oplus} \mid \boldsymbol{B}_{\oplus}^{*}, \Omega_{\oplus}\right)=\frac{1}{\sqrt{2 \pi} \sigma} \times \\
\exp \left[-\frac{\left(\boldsymbol{\lambda}^{* T} \boldsymbol{B}_{\oplus}^{*}-\left(\Omega_{\oplus}-\boldsymbol{\lambda}^{T} \boldsymbol{B}_{\oplus}\right)\right)^{2}}{2 \sigma^{2}}\right],
\end{aligned}
$$

where $\boldsymbol{B}_{\oplus}^{(*)}$ is either $\boldsymbol{B}_{t}^{(*) \mathrm{MP}}-\boldsymbol{B}_{t}^{(*) \mathrm{PF}}$ or $\boldsymbol{B}_{n}^{(*)}$ depending on whether we are interested in the tangential or normal component, respectively. Likewise, the value of $\Omega_{\oplus}$ is $\mu_{0} I_{p}$ for the tangential component or simply 0 for the normal component. $\sigma$ is the noise standard deviation based on the measured magnetic signals with the uncertainty propagation, and measured to be $\mathcal{O}\left(10^{-4}\right)$.

Finally, we obtain posterior as

$$
p\left(\boldsymbol{B}_{\oplus}^{*} \mid \boldsymbol{B}_{\oplus}, \Omega_{\oplus}\right) \propto p\left(\boldsymbol{B}_{\oplus} \mid \boldsymbol{B}_{\oplus}^{*}, \Omega_{\oplus}\right) p\left(\boldsymbol{B}_{\oplus}^{*} \mid \Omega_{\oplus}\right),
$$

providing us inferred values of the missing magnetic signals $\left(\boldsymbol{B}_{\oplus}^{*}\right)$ consistent with the measured signals $\left(\boldsymbol{B}_{\oplus}\right.$ and $\sigma$ ) and the Maxwell's equations $\left(\Omega_{\oplus}\right)$ assuming that $p\left(\Omega_{\oplus}\right)=1$. With a uniform prior $p\left(\boldsymbol{B}_{\oplus}^{*} \mid \Omega_{\oplus}\right)$, it is obvious that we obtain infinite number of solutions from maximum a posteriori (MAP) method if we have more than one unknown of the same component. In simpler words, we have only one equation for the tangential (Ampère's law) or the normal (Gauss's law for magnetism) component; thus, more than one unknown of the same component results in infinite number of solutions. Fig. 2 shows an estimated log-posterior distribution, $\ln \left[p\left(\boldsymbol{B}_{\oplus}^{*} \mid \boldsymbol{B}_{\oplus}, \Omega_{\oplus}\right)\right]$, where we have removed two $B_{t}$ measurements, i.e., probe numbers \#15 and \#16, and confirms this effect clearly as depicted by the thick black line corresponding to the MAPs. This is the limitation of the imputation scheme solely based on the Bayes' model consistent with the Maxwell's equations. 


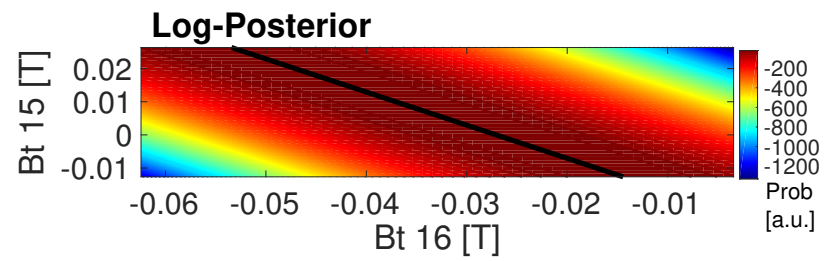

FIG. 2: Log-posterior, $\ln \left[p\left(\boldsymbol{B}_{\oplus}^{*} \mid \boldsymbol{B}_{\oplus}, \Omega_{\oplus}\right)\right]$, of the missing magnetic signals inferred by the Bayes' model with the Maxwell's equations, when two tangential components $\left(B_{t}\right.$ from MPs \#15 and \#16) of the magnetic signals are missing. Thick black line marks where the posterior is maximum indicating that infinite number of solutions are possible. Data are inferred for KSTAR shot \#9010 at $0.1 \mathrm{sec}$.

\section{B. Based on Gaussian Process}

Motivated by the limitation of the Baye's model with the Maxwell's equations, we introduce Gaussian Process ${ }^{5}$ (GP) in our imputation scheme. We express the probability distribution of $\boldsymbol{B}^{*}\left(N_{m} \times 1\right.$ column vector $)$ given the measured data $\boldsymbol{B}\left(N_{i} \times 1\right.$ column vector $)$ without any analytic expression of the data a priori as described elsewhere $^{5,15}$

$$
p\left(\boldsymbol{B}^{*} \mid \boldsymbol{B}\right)=\mathcal{N}\left(\underline{\underline{\boldsymbol{K}}}^{*} \underline{\underline{\boldsymbol{K}}}^{-1} \boldsymbol{B}, \underline{\underline{\boldsymbol{K}}}^{* *}-\underline{\underline{\boldsymbol{K}}}^{*} \underline{\underline{\boldsymbol{K}}}^{-1} \underline{\underline{\boldsymbol{K}}}^{* T}\right),
$$

with

$$
\begin{array}{ll}
\underline{\underline{\boldsymbol{K}}} \equiv \underline{\underline{\boldsymbol{K}}}(\boldsymbol{X}, \boldsymbol{X})+\sigma_{n}^{2} \underline{\underline{\boldsymbol{I}}}, & \left(N_{i} \times N_{i} \text { matrix }\right) \\
\underline{\underline{\boldsymbol{K}}}^{*} \equiv \underline{\underline{\boldsymbol{K}}}\left(\boldsymbol{X}^{*}, \boldsymbol{X}\right), & \left(N_{m} \times N_{i} \text { matrix }\right) \\
\underline{\underline{\underline{K}}}^{* *} \equiv \underline{\underline{\boldsymbol{K}}}\left(\boldsymbol{X}^{*}, \boldsymbol{X}^{*}\right), & \left(N_{m} \times N_{m} \text { matrix }\right)
\end{array}
$$

where $\mathcal{N}($, ) is the usual notation for a normal distribution, and $\underline{\underline{I}}$ the identity matrix. $\sigma_{n}^{2} \sim \mathcal{O}\left(10^{-4}\right)$ is determined by treating it as a hyperparameter for the numerical stability during matrix inversion. ${ }^{5,16,17}$ Recall that $N_{i}\left(N_{m}\right)$ is the total number of intact (missing) magnetic signals. Here, $\boldsymbol{X}^{(*)}$ is the $2 \times N_{i}\left(N_{m}\right)$ matrix containing the physical positions of all the intact (missing) magnetic probes in two dimensional space, i.e., physical $R$ and $Z$ positions at a fixed toroidal location.

The $i^{\text {th }}$ and $j^{\text {th }}$ component of a covariance matrix $\underline{\underline{\boldsymbol{K}}}^{(* \text { or **)}}$ is defined as

$$
\begin{aligned}
& K_{i j}^{(* \text { or } * *)}\left(\boldsymbol{x}_{i}^{(*)}, \boldsymbol{x}_{j}^{(*)}\right)= \\
& \sigma_{f}^{2} \exp \left[-\frac{1}{2}\left(\boldsymbol{x}_{i}^{(*)}-\boldsymbol{x}_{j}^{(*)}\right)^{T}\left[\begin{array}{cc}
\ell_{R}^{2} & 0 \\
0 & \ell_{Z}^{2}
\end{array}\right]^{-1}\left(\boldsymbol{x}_{i}^{(*)}-\boldsymbol{x}_{j}^{(*)}\right)\right],
\end{aligned}
$$

where $\boldsymbol{x}_{i}^{(*)}$ is the $i^{\text {th }}$ column vector of the $\boldsymbol{X}^{(*)}$, i.e., $2 \times 1$ column vector containing the physical positions of the $i^{\text {th }}$ magnetic probe in $R$ and $Z$ coordinate. Hyperparameters $\sigma_{f}^{2}, \ell_{R}$ and $\ell_{Z}$ are the signal variance and the length (a)

(c)

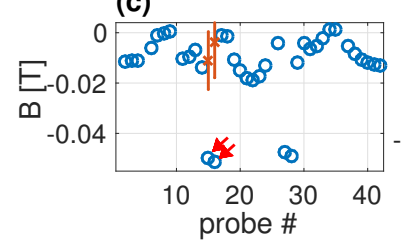

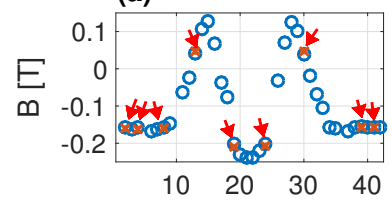

(b)
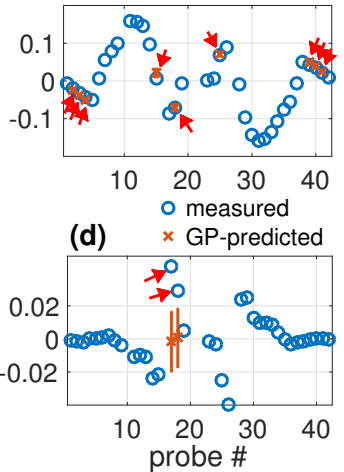

FIG. 3: Successful GP predictions (red crosses) compared with the actual data (blue circles) for (a) $B_{t}$ and (b) $B_{n}$ at $3.70 \mathrm{sec}$ of KSTAR shot \#9010 where we remove nine non-consecutive signals (indicated by red arrows) simultaneously to examine the proposed GP imputation scheme. On the other hand, if the magnetic signals are spatially varying fast such as (c) $B_{t}$ of MPs \#15 and \#16 and (d) $B_{n}$ of MPs \#17 and \#18 at $0.10 \mathrm{sec}$ of the same shot, the GP imputation scheme fails to infer the correct values.

scales in $R$ and $Z$ directions, respectively. These hyperparameters govern the characteristic of the Gaussian process, i.e., Eq. (6), and we select the hyperparameters such that the evidence $p(\boldsymbol{B})$ is maximized ${ }^{18}$ with an assumption ${ }^{19}$ of $\ell_{R}=\ell_{Z}$ for simplicity. As searching for the hyperparameters may become time consuming, thus not applicable for real-time control, one can obtain these values beforehand using many existing plasma discharges as for the case of density reconstruction. ${ }^{16}$ Once we have values for the hyperparameters, i.e., $\sigma_{f} \sim \mathcal{O}\left(10^{-2}\right)$ and $\ell_{R}=\ell_{Z} \sim \mathcal{O}\left(10^{-1}\right)$ in this study, we use Eq. (6) to obtain the values of the missing magnetic signals $\boldsymbol{B}^{*}$, i.e., $\boldsymbol{B}^{*}=\boldsymbol{K}^{*} \boldsymbol{K}^{-1} \boldsymbol{B}$.

Fig. $3(\mathrm{a})$ and (b) show that our proposed GP imputation scheme successfully infers the missing magnetic signals both for (a) $B_{t}$ and (b) $B_{n}$ where the red crosses are the inferred values and the blue circles are the measured (actual) values. We have examined our scheme with up to nine non-consecutive missing signals indicated by the red arrows.

We have also found that the GP imputation scheme fails to infer the correct values if the magnetic signals are varying fast in space as shown in Fig. 3(c) for $B_{t}$ and (d) for $B_{n}$. This is the limitation of the GP-only imputation scheme.

\section{Based on Bayes' model coupled with Gaussian Process}

As we find the limitations of the Bayes' model (infinite number of solutions for more than one missing magnetic signal) and the GP (incorrect inference for spatially fastvarying missing magnetic signals), we resolve such weak- 
nesses by combining the two schemes: for instance, if we have seven missing signals, we select one missing signal among the seven. Then, we use the GP to infer the non-selected six missing ones based on the intact signals together with the selected missing one which is inferred based on the Bayes' model.

Let us denote the selected missing magnetic signal as $B_{k}^{*}$, and define $\check{\boldsymbol{X}}^{*}$ to contain the positions of $R$ and $Z$ for all the missing magnetic signals except the ones corresponding to $B_{k}^{*}$ resulting in $2 \times\left(N_{m}-1\right)$ matrix; while $\check{\boldsymbol{X}}$ containing those of $B_{k}^{*}$ in addition to intact magnetic signals becoming $2 \times\left(N_{i}+1\right)$ matrix, i.e., concatenate those of $B_{k}^{*}$ at the last column of $\boldsymbol{X}$. With $\check{\boldsymbol{X}}^{*}$ and $\check{\boldsymbol{X}}$ our covariance matrices become

$$
\begin{aligned}
& \underline{\underline{\boldsymbol{K}}} \equiv \underline{\underline{\boldsymbol{K}}}(\check{\boldsymbol{X}}, \check{\boldsymbol{X}})+\sigma_{n}^{2} \underline{\underline{\boldsymbol{I}}},\left(\left(N_{i}+1\right) \times\left(N_{i}+1\right) \text { matrix }\right) \\
& \stackrel{\check{\boldsymbol{K}}}{ }^{*} \equiv \underline{\underline{\boldsymbol{K}}}\left(\check{\boldsymbol{X}}^{*}, \check{\boldsymbol{X}}\right), \quad\left(\left(N_{m}-1\right) \times\left(N_{i}+1\right) \text { matrix }\right) \\
& \underline{\underline{\boldsymbol{K}}}^{* *} \equiv \check{\underline{\boldsymbol{K}}}\left(\check{\boldsymbol{X}}^{*}, \check{\boldsymbol{X}}^{*}\right), \quad\left(\left(N_{m}-1\right) \times\left(N_{m}-1\right) \text { matrix }\right)
\end{aligned}
$$

We separate out the last column of the $\left(N_{m}-1\right) \times\left(N_{i}+1\right)$ matrix of $\check{\underline{\boldsymbol{K}}}^{*} \underline{\underline{\boldsymbol{K}}}^{-1}$ containing $B_{k}^{*}$ information and denote this column vector as $\boldsymbol{L}$ and the rest of the matrix, i.e., without the last column of $\underline{\underline{\underline{K}}}^{*} \underline{\underline{\boldsymbol{K}}}^{-1}$, be $\underline{\underline{\Lambda}}$. Since we have found that $\boldsymbol{B}^{*}=\underline{\underline{K}}^{*} \underline{\underline{K}}^{-1} \boldsymbol{B}$ in Sec. II B, we obtain

$$
\check{\boldsymbol{B}}_{\oplus}^{*}=\underline{\underline{\Lambda}} \boldsymbol{B}_{\oplus}+\boldsymbol{L} B_{k \oplus}^{*},
$$

stating that once $B_{k}^{*}$ is determined, then all the other missing magnetic signals $\check{\boldsymbol{B}}^{*}$ are determined by the GP. We find the unknown $B_{k}^{*}$ using the Bayes' model where it is perfectly applicable since we have only one missing signal as discussed in Sec. II A. Thus, $\boldsymbol{\lambda}^{* T} \boldsymbol{B}_{\oplus}^{*}$ in Eq. (4) is

$$
\begin{aligned}
\boldsymbol{\lambda}^{* T} \boldsymbol{B}_{\oplus}^{*} & =\lambda_{k}^{*} B_{k \oplus}^{*}+\check{\boldsymbol{\lambda}}^{* T} \check{\boldsymbol{B}}_{\oplus}^{*} \\
& =\left(\lambda_{k}^{*}+\check{\boldsymbol{\lambda}}^{* T} \boldsymbol{L}\right) B_{k \oplus}^{*}+\check{\boldsymbol{\lambda}}^{* T} \underline{\underline{\Lambda}} \boldsymbol{B}_{\oplus},
\end{aligned}
$$

where $\lambda_{k}^{*}$ and $\check{\lambda}^{*}$ are the segment distances for the selected missing one $B_{k \oplus}^{*}$ and for the rest of the missing signals, respectively.

Slightly modifying Eq. (4) to include the GP scheme, our likelihood for the Bayes' model, then, becomes

$$
\begin{aligned}
p\left(\boldsymbol{B}_{\oplus} \mid B_{k \oplus}^{*}, \Omega_{\oplus}\right) & =\frac{1}{\sqrt{2 \pi} \sigma} \exp \left[\frac { 1 } { 2 \sigma ^ { 2 } } \left(\left(\lambda_{k}^{*}+\check{\boldsymbol{\lambda}}^{* T} \boldsymbol{L}\right) B_{k \oplus}^{*}\right.\right. \\
& \left.\left.-\left(\Omega_{\oplus}-\boldsymbol{\lambda}^{T} \boldsymbol{B}_{\oplus}-\check{\boldsymbol{\lambda}}^{* T} \underline{\underline{\Lambda}} \boldsymbol{B}_{\oplus}\right)\right)^{2}\right] .(10
\end{aligned}
$$

The likelihood now contains only one unknown $B_{k}^{*}$, and all the rest of the missing signals are treated as known ones using the GP, i.e,. Eq. (8).

We construct the prior $p\left(B_{k \oplus}^{*} \mid \Omega_{\oplus}\right)$ to follow a Gaussian distribution with the mean of $B_{k \oplus}^{\text {pair }}$ and the variance of $\sigma_{\text {prior }}^{2} . B_{k \oplus}^{\text {pair }}$ is the signal of the magnetic probe from the up-down symmetric position of the missing signal $B_{k \oplus}^{*}$. MPs \#6 and \#37, MPs \#12 and \#31, and MPs \#19 and \#24 in Fig. 1(a) are examples. We use such a paired magnetic signal as a prior mean of the missing signal because KSTAR discharges are quite up-down symmetric, so that a typical correlation between the paired signals is about 0.9 . Regarding the prior variance $\sigma_{\text {prior }}^{2}$, to minimize possible biases we set it to be 500 which means that the prior distribution is largely uniform since the actual values of the magnetic signals are not much larger than $0.1 \mathrm{~T}$ as shown in Fig. 3 .

We finally obtain the posterior following Eq. (5) as

$$
\begin{aligned}
p\left(B_{k \oplus}^{*} \mid \boldsymbol{B}_{\oplus}, \Omega_{\oplus}\right) \propto \\
\quad \exp \left[-\frac{\left(B_{k \oplus}^{*}-B_{k \oplus}^{\star}\right)^{2}}{2 \sigma_{\mathrm{GP}}^{2}}-\frac{\left(B_{k \oplus}^{*}-B_{k \oplus}^{\text {pair }}\right)^{2}}{2 \sigma_{\text {prior }}^{2}}\right],
\end{aligned}
$$

where

$$
\begin{aligned}
B_{k \oplus}^{\star} & =\frac{\Omega_{\oplus}-\boldsymbol{\lambda}^{T} \boldsymbol{B}_{\oplus}-\check{\boldsymbol{\lambda}}^{* T} \underline{\underline{\Lambda}} \boldsymbol{B}_{\oplus}}{\lambda_{k}^{*}+\check{\boldsymbol{\lambda}}^{* T} \boldsymbol{L}} \\
\sigma_{\mathrm{GP}}^{2} & =\left[\frac{\sigma}{\lambda_{k}^{*}+\check{\boldsymbol{\lambda}}^{* T} \boldsymbol{L}}\right]^{2} .
\end{aligned}
$$

Thus, maximum a posteriori (MAP) denoted as $B_{k \oplus}^{\mathrm{MAP}}$ can be analytically estimated and is

$$
B_{k \oplus}^{\mathrm{MAP}}=\left[\frac{B_{k \oplus}^{\star}}{\sigma_{\mathrm{GP}}^{2}}+\frac{B_{k \oplus}^{\mathrm{pair}}}{\sigma_{\text {prior }}^{2}}\right]\left[\frac{1}{\sigma_{\mathrm{GP}}^{2}}+\frac{1}{\sigma_{\text {prior }}^{2}}\right]^{-1}
$$

with the posterior variance $\sigma_{\text {post }}^{2}=\left(1 / \sigma_{\mathrm{GP}}^{2}+1 / \sigma_{\text {prior }}^{2}\right)^{-1}$. Once $B_{k \oplus}^{\mathrm{MAP}}$ is found, then all the other missing signals are determined by Eq. (8). This completes the imputation process.

To validate our proposed imputation scheme based on the Bayes' model coupled with the GP, we take the same examples shown in Fig. 3(c) and (d). Fig. 4(a) $B_{t}$ from MPs \#15 and \#16 and (b) $B_{n}$ from MPs \#17 and \#18 show considerable improvements where the green triangles inferred by the Bayes' model coupled with the GP are very close to the blue circles which are the measured values. Again, the red crosses obtained only by the GP fails to do so.

Fig 4(c) $B_{t}$ from MP \#15 and (d) $B_{n}$ from MP \#17 from KSTAR shot \#9427 show temporal evolutions of the inferred values where the blue line is the measured values, the red line for the GP only and the green line for the Bayes' model with the GP. Typically, the GPonly method fails largely during ramp-up and ramp-down phases while it is not too bad during the flat-top phase; whereas the Bayes' model with the GP finds the correct values throughout the whole discharge.

Eq. (12) contains no unknowns which means that $B_{k \oplus}^{\mathrm{MAP}}$ can be estimated in real-time. In fact, our proposed method takes less than $1 \mathrm{msec}$ on a typical personal computer. The hyperparameters are prepared beforehand based on many previous discharges, and missing or faulty signals can be identified ${ }^{6,7}$ in real-time. What one requires to do is simply to perform the following 
(a) $\circ$ Measured $\times$ GP $\triangle$ GP-Bayes (b)

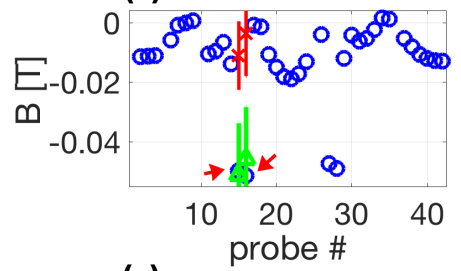

(c)
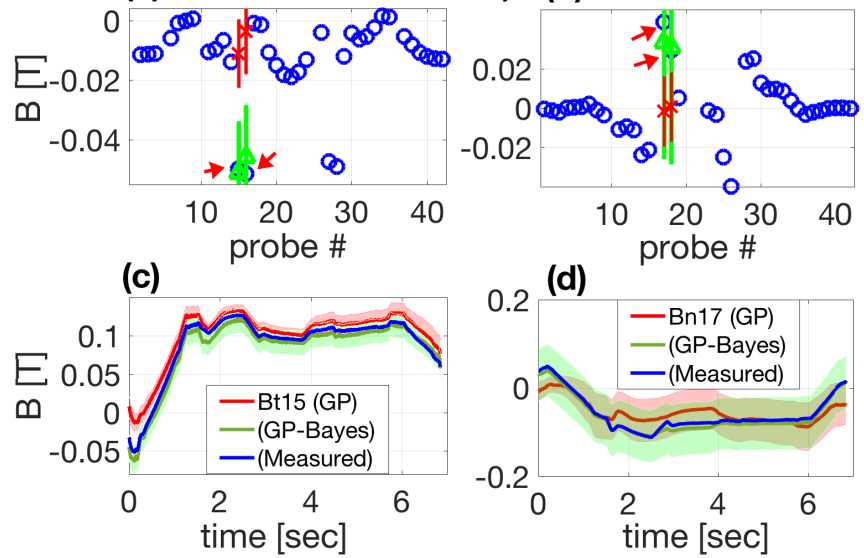

(d)

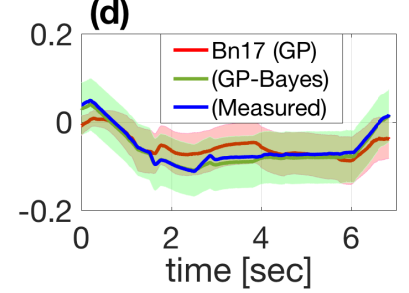

FIG. 4: (a) $B_{t}$ from MPs $\# 15$ and \#16 and (b) $B_{n}$ from MPs \#17 and \#18 from KSTAR shot \#9010 at $0.1 \mathrm{sec}$ as shown in Fig. 3(c) and (d). Green triangles obtained by the Bayes' mode with the GP match the measured values (blue circles) well, while the GP-only method (red crosses) fails to do so as has been discussed in Sec. II B. Comparisons of temporal evolutions for (c) $B_{t}$ from MP \#15 and (d) $B_{n}$ from MP \#17 from KSTAR shot \#9427 where blue line is the measured values, red line for the GP-only and green line for the Baye's model with the GP. Green lines agree well with blue lines well throughout the whole discharge including ramp-up and ramp-down phases.

three steps in real-time: $(1)$ select a missing signal $\left(B_{k \oplus}^{*}\right)$ among all the missing ones $\left(\boldsymbol{B}_{\oplus}^{*}\right),(2)$ estimate noise levels $(\sigma)$ of the measured signals and (3) apply Eq. (12) and Eq. (8) to impute more than one missing magnetic signals. Good choice of a missing signal $\left(B_{k \oplus}^{*}\right)$ is from the ones that spatially vary fast if they exist. In KSTAR such signals are $B_{t}$ from MPs \#15 and \#16, and $B_{n}$ from MP \#17 and \#18 in almost all cases, if not all.

\section{CONCLUSION}

We have developed and presented a real-time inference scheme, thus imputation scheme, for missing or faulty magnetic signals. Our method, Bayes' model with the likelihood constructed based on Gauss's law for magnetism and Ampère's law, coupled with the Gaussian process, allows one to infer the correct values even if more than one missing signal that is spatially varying fast exists. The coupled method outperforms the Baye'sonly and the GP-only methods without losing their own advantages. We have examined our method up to nine non-consecutive missing magnetic signals.

The proposed method takes less than $1 \mathrm{msec}$ on a typical personal computer, so that the method can be applied to fusion-grade plasma operations where real-time reconstruction of magnetic equilibria is crucial. It can also be used for a neural network trained with a complete set of magnetic signals without fearing the possible loss of magnetic signals during plasma operations.

As a possible future work, developing a real-time searching algorithm for the hyperparameters in the Gaussian process that optimizes the evidence will be beneficial. Although results with the predetermined hyperparameters based on many previous discharges can be satisfying, the hyperparameters specific to a current discharge may provide much better plasma controls especially for those discharges that we have not yet explored much. In addition, including the effect of eddy currents can improve the performance of our method especially during the ramp-up and down phases and disruptions.

\section{ACKNOWLEDGEMENT}

This research was supported by National R\&D Program through the National Research Foundation of Korea (NRF) funded by the Ministry of Science and ICT (grant number NRF-2017M1A7A1A01015892 and NFR2017R1C1B2006248) and the KUSTAR-KAIST Institute, KAIST, Korea.

\section{REFERENCES}

${ }^{1}$ J. B. Lister and H. Schnurrenberger, Nuclear Fusion 31, 1291 (1991).

${ }^{2}$ E. Coccorese, C. Morabito, and R. Martone, Nuclear Fusion 34, 1349 (1994).

${ }^{3}$ J. W. C. van Lint et al., Transportation Research Part C: Emerging Technologies 13, 347 (2005).

${ }^{4}$ D. S. Sivia and J. Skilling, Data Analysis: A Bayesian Tutorial (Oxford: Oxford University Press, 2006).

${ }^{5}$ C. E. Rasmussen and C. K. I. Williams, Gaussian Processes for Machine Learning (The MIT Press, 2006).

${ }^{6}$ A. C. Neto, D. Alves, B. B. Carvalho, et al., IEEE Transactions on Nuclear Science 61, 1228 (2014).

${ }^{7}$ R. Nouailletas, P. Moreau, and S. Bremond, Fusion Engineering and Design 87, 289 (2012).

${ }^{8}$ M. Kwon et al., Nuclear Fusion 51 (2011).

${ }^{9}$ L. L. Lao, H. S. John, R. D. Stambaugh, A. G. Kellman, and W. Pfeiffer, Nuclear Fusion 25, 1611 (1985).

${ }^{10}$ J. R. Ferron, M. L. Walker, L. L. Lao, H. E. S. John, D. A. Humphreys, and J. A. Leuer, Nuclear Fusion 38, 1055 (1998).

${ }^{11}$ S. G. Lee, J. G. Bak, E. M. Ka, J. H. Kim, and S. H. Hahn, Review of Scientific Instruments 79, 10F117 (2008).

12 J. Freidberg, Ideal MHD (Cambridge University Press, 2014).

${ }^{13} \mathrm{~S}$. Tsaun and H. Jhang, Fusion Engineering and Design 82, 163 (2007).

${ }^{14}$ J. M. Moret, F. Buhlmann, D. Fasel, F. Hofmann, and G. Tonetti, Review of Scientific Instruments 69, 2333 (1998).

${ }^{15}$ R. von Mises, Mathematical Theory of Probability and Statistics (Academic Press, 1964).

${ }^{16}$ S. Kwak, J. Svensson, M. Brix, and Y.-c. Ghim, Nuclear Fusion 57 (2017).

${ }^{17}$ L. Foster et al., Journal of Machine Learning Research 10, 857 (2009).

${ }^{18}$ S. Kwak, J. Svensson, M. Brix, Y.-c. Ghim, and JET Contributors, Review of Scientific Instruments 87, 023501 (2016).

${ }^{19}$ D. Li, J. Svensson, H. Thomsen, F. Medina, A. Werner, and R. Wolf, Rev. Sci. Instrum. 84, 083506 (2013). 